\title{
APOCYNACEAE TÓXICAS E MEDICINAIS OCORRENTES NOS ESTADOS DE PERNAMBUCO E PARAÍBA, BRASIL
}

\author{
Maria Dulce Belo de Moura (1) \\ Maria de Fátima Agra (2)
}

\begin{abstract}
RESUMO - É apresentada uma lista de 37 espécies da família Apocynaceae de Pernambuco e da Paraíba, Brasil, pela análise de dados da literatura e dos exemplares existentes nos herbários destes Estados. São fornecidas informações etnofarmacológicas de 14 espécies, bem como uma lista dos constituintes com atividades farmacológicas de 12 espécies.
\end{abstract}

Palavras-chave: Apocynaceae, etnofarmacologia, plantas tóxicas e medicinais, flora do NE do Brasil.

\begin{abstract}
In this paper are listed 37 Apocynaceae species from Pernambuco and Paraiba, Brazil. The informations were obtained from herbarium sheets. Ethnopharmacological uses of 14 species and the results of a literature review are also reported. Medical uses, known constituents and pharmalogical are presented for 12 species.
\end{abstract}

Key words: Apocynaceae, ethnopharmacology, toxic and medicinal plants, flora of NE Brazil.

\section{Introdução}

As Apocynaceae constituem uma família que se caracteriza por apresentar grande variabilidade morfológica em seus órgãos florais, presença de vasos laticíferos e pela diversidade de substâncias resultantes do seu metabolismo secundário que, na maioria das vezes, possuem propriedades farmacológicas. Economicamente, também são bastante utilizadas na ornamentação de parques e

(1) Mestrado em Botânica, UFRPE, Rua D. Manoel de Medeiros s/n, Dois Irmãos, 50.000 - Recife - PE. Bolsista do CNPq.

(2) Laboratório de Tecnologia Farmacêutica, UFPb, Cx. Postal 5009, 58.059 - João Pessoa-PB. Bolsista da CAPES. Solicitação de separatas. 
jardins, principalmente as especies pertencentes aos gêneros: Catharanthus, Nerium, Plumeria e Thevetia.

A família consiste de cerca de 200 gêneros a 2000 espécies, distribuídas nas regiōes tropicais e, eventualmente, em clima temperado, sendo representado, no Brasil, por 41 gêneros com 376 espécies, aproximadamente.

A posição taxonômica das Apocynaceae em relação às outras famílias da mesma ordem, Gentianales, é colocada por CRONQUIST (1981), próxima das Asclepiadaceae pela presença de vasos laticíferos e de glicosídeos cardiotônicos existentes, que não são encontrados nas outras famílias da mesma ordem. A classificação infrafamiliar é bastante discutida e várias divisões para a família, foram propostas (WOODSON, 1939; PICHON, 1948; ALLORGE, 1980), baseadas nas características das estruturas florais. Estudos quimiotaxonômicos também têm sido realizados, para um maior conhecimento da sistemática das Apocynaceae, que apresentam sua heterogeneidade mais marcada a nível da subfamília Pumerioideae (GOTTLIEB, 1982). Cerca de 400 alcalóides já foram descritos, dos quais 260 com a estrutura química elucidada (RAFFAUF, 1964).

Reconhecendo a importância das Apocynaceae e a necessidade de maiores informações sobre seus representantes nos Estados de Pernambuco e Paraíba, efetuou-se o levantamento do material de herbário coletado nestes Estados, e o estudo comparativo na bibliografia especializada das espécies reputadas por suas propriedades tóxicas e medicinais.

\section{Material e Métodos}

A pesquisa foi realizada em duas etapas. Na primeira, foram analisadas um total de 226 exsicatas, depositadas nos herbários da Empresa Pernambucana de Pesquisa Agropecuária (IPA); Prof. João Vasconcelos Sobrinho (PEUFR), Universidade Federal Rural de Penuambuco; Universidade Federal de Pernambuco (UFP) e Prof. Lauro Pires Xavier (JPB) da Universidade Federal da Paraŕba. Para o levantamento das espécies e informações referentes ao uso etnobotânico, o material foi identificado a nível de espécie, quando possível. Na segunda etapa, foi realizada a pesquisa bibliográfica das espécies referidas, por seu emprego na medicina popular ou por suas propriedades tóxicas.

\section{Resultados e Discussão}

A tabela 1 apresenta, em ordem alfabética por gênero, a lista das espécies coletadas nos Estados de Pernambuco e Paraíba, nas diferentes zonas fitogeográficas classificadas por VASCONCELOS SOBRINHO (1949).

$\mathrm{Na}$ tabela 2 estão relacionados as espécies e uso(s) etnofarmacológico na região, com dados referentes à nomenclatura e parte(s) usada(s). Os dados referentes aos constituintes, atividades farmacológicas e fonte bibliográfica, encontram-se na tabela 3.

O total de espécies identificadas foi de 37, subordinadas a 19 gêneros, sendo Aspidosperma o gênero mais representado com 6 espécies, consideradas 
tóxicas, porém sem emprego na medicina popular. Das espécies inventariadas, apenas $14(38 \%)$ apresentam informações do uso etnofarmacológico e 12 (35\%) são referidas por suas atividades farmacológicas já comprovadas, inclusive a espécie Macoubea guianensis Aubl., que não é referida popularmente, mas possui atividade curarizante (HAMEL \& HAMEL, 1988b). As espécies Hancornia speciosa Gomes e Mandevilla scabra R. et S. são referidas por MONACHINO (1945) e AZAMBUJA (1947) por suas propriedades medicinais, mas não apresentam informações sobre sua farmacologia.

Catharanthus roseo (L.) G. Don., que cresce subespontaneamente no nordeste, é a espécie que apresentou o maior número de referências sobre os constituintes isolados e suas atividades farmacológicas comprovadas, destacando-se a vincristina e a vinblastina, usadas no tratamento da leucemia, comercializadas sob os nomes de Velban e Oncovim (CALABRESI \& PARKS, 1973).

\section{Agradecimentos}

As autoras agradecem ao Dr. Marcel Plumel, Laboratoire de Phanerogamie, Museum National d'Histoire Naturelle, Paris, France, por sua prestimosa ajuda com a bibliografia utilizada; ao CNPq e à CAPES, pelo apoio financeiro.

\section{Referências Bibliográficas}

ALLORGE, L., HUSSON, H.P. \& SASTRE, C. 1981. Morphologie et chimiotaxomie des Apocynacées. Conclusions phylogenetiques et biogeographiques. C. R. Biogeogr. 57(3): 112-126.

AZAMBUJA, D. 1947. Contribuição ao conhecimento das Apocynaceae encontradas no Brasil. Arq. Serv. Flor. Rio de Janeiro 3: 11-112.

CALABRESI, P. \& PARKS, R.E., Jr. 1973. Quimioterapia das doenças neoplásicas. In: GOODMAN, L.S. \& GILMAN, A. (eds.) As bases farmacológicas da terapêutica. 4 ed.: 1270-1274. Rio de Janeiro, Guanabara Koogan.

CHOPRA, R.N., NAYAR, S.L. \& CHOPRA, S.I. 1956. Glossary of Indian medicinal plants. C.S.I.R. New Delhi.

CRONQUIST, A. 1981. An integrated system of classification of flowering plants. Columbia University. New York.

GROMAN, M., TUST, R,H,, SVOBODA, G.H. \& LE MEN, J. 1964. Alkaloids of Vinca rosea (Catharanthus roseus). XXVI. Structure activity studies of some alkaloids and their derivaties. Lloydia 27(3): 214-219.

GOTTLIEB, O.R. 1982. Evolution of indole alkaloids in Apocynaceae. In: Micromolecular evolution, systematics and ecology. Springer ed. Berlin, New York: 71-79.

HAMEL, M.C. \& HAMEL, J.L. 1988a. Contribuition bibliographique a la conaissance botanique des Apocynacées. III Données phytochimiques. 1. Derives de l'iridodial, cardenolides, alcaloides steroidiques. Rev. Cytol. Biol. veget. (Bot.) 11: 123-144.

HAMEL, M.C. \& HAMEL, J.L. 1988b. Contribuition bibliographique a la conaissance botanique des Apocynacées. III Donées phytochimiques. 2. Alcaloides indoliques. Conclusions. Rev. Cytol. Biol. veget. (Bot.) 11: 145-191.

HEGNAUER, R. 1964. Apocynaceae in chemotaxonomie der Pflanzen. t. e Dicotiledoneae, I. Birkhauser. Bale et Stuttgart: 124-163. 
JOHNSON, I.S., WRIGHT, H.F. \& SVOBODA, G.H. 1959. Experimental basis for clinical evolution of anti-tumor principles derived from Vinca rosea L. J. Lab. Clin. Med. 54: 830 .

GORMAN, M., TULST, R.H., SVOBODA, G.H. \& LE MEN. J. 1964. aLKALOIDS OF Vinca rosea (Catharanrhus roseus). XXWI. Structure-activity studies some alkaloids and their derivaties. Lloydia 27(3): 214-219.

MORAIS, M. do S. QUEIROZ. 1983. Estudos fitoquímicos e ensaios farmacológicos preliminares de Allamanda blanchetii A. DC. Dissertação de mestrado. Universidade Federal da Paraíba. João Pessoa.

MORS, W.B., SALTZMAN, P., BEEREBOOM, J., PAKRASHI, S.C., DJERASSI, C. 1956. Alkaloids the two Brazilian Apocynaceae: Rauvolfia grandiflora Mart. and Lochnera (Vinca Rosea L.) Reichb. var. alba (Sweet) Herbbd. Cuem. Ind. (London): 173-174.

MONACHINO, J. 1945. A revision of Hancornia (Apocynaceae). Lilloa 11: 28-48.

PICHON, M. 1948. Classification des Apocynacées: IX. Rauvolfiées, Alstoniées, Allamandées et Tabernaemontanoidées. Mem. Mus. Natl. Hist. Nat. 27(6): 153-251.

RAFFAUF, R.E. 1964. Some chemiotaxonomic considerations in the Apocynaceae. Lloydia 27(4): 287-333.

SVOBODA. G.H., NEUSS, N. \& GORMAN, M. 1959. Alkaloids of Vinca rosea Linn. (Catharanthus roseus G. Don.). V. Preparation and characterization of alkaloids. $J$. Am. Pharm. Assoc. 48: 659-666.

SVOBODA, G.H., GORMAN, M. \& TUST, R.H. 1964. Alkaloids of Vinca rosea (Catharanthus roseus). XKKV. Lochrovine, Perivimivine, Vincoline, Lochrovidine, Lochrovicine and Vincolidine. Lloydia 27(3): 203-213.

VASCONCELOS SOBRINHO, J. de. 1949. As regiōes naturais de Pernambuco, o meio e a civilização. Publ. no 2. Inst. Pesq. Agron. Pernambuco, Recife.

WANDERLEI, M. de. F. 1985. Constituintes químicos e estudos farmacológicos esperimentais de Himathanthus phagedaenicus (Mart.) Woodson. Dissertação de mestrado. Universidade Federal da Paraíba. João Pessoa.

WATT, J.M. \& BREYER-BRANDWIJK, M.G. 1962. The medicinal and poisonous plants of southern and eastern Africa. 2 ed. London: E. \& Livingstone.

WOODSON, R.E., Jr. 1939. Studies in the apocynaceae. I. A critical study of the Apocynaceae (with special reference tothe genus Apocynum). Ann. Missouri Bot. Gard. 17: $1-212$.

WOODSON, R.E., Jr., YOUNKEN, H.W., SCHLITTLER, E. \& SCHNEIDER, J.A. 1957. Rauwolfia: botany, pharmacognosy, chemistry \& pharmacology. Boston: Brown \& Company. 
Tabela 1 - Apocynaceae de Pernabuco e da Paraíba e sua distribuição fitogeográfica.

\section{ESPÉCIES}

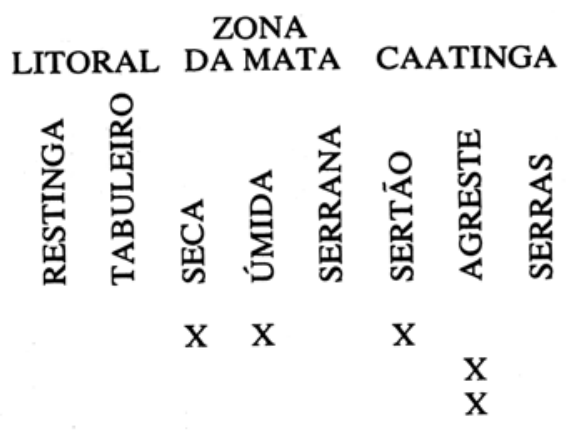

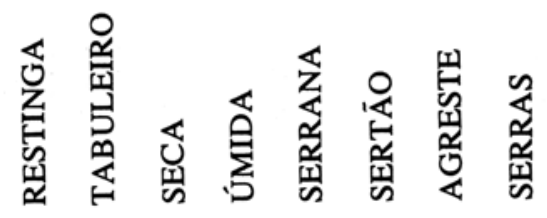

Allamanda puberula A.DC.

Allamanda blanchetii A.DC.

Allamanda violacea Gardn.

*Allamanda carthartica $\mathrm{L}$.

Arduina bispinosa L.

Aspidosperma discolor A.DC.

Aspidosperma limae Wood.

Aspidosperma pyrifolium Mart.

Aspidosperma nigricans Hamdro.

Aspidosperma pyricollum M. Arg.

Aspidosperma refractum Mart.

$\mathrm{X}$

$\mathrm{X} \quad \mathrm{X}$

* Catharanthus roseus L.G.Don.

Echites varia (Stdm.)M.Arg.

Echites violacea Vell.

*Ervatamia coronaria (Jacq.) Stapf.

Forsteronia glabrescens M.Arg.

Forsteronia leptocarpa (Hook. et. Art.)A.DC.

Hancornia speciosa Gomez.

Himathanthus phadedaenicus (Mart.)Woodson

Macoubea guianesis Aubl.

Mandevilha funiformis (Vell.)K. Schum.

Mandevilha ilustris (Vell.)Woodson

Mandevilha moricandiana (DC.) Woodson

Mandevilha scabra (Roem.et.Schult.)K.Sch.

*Nerium oleander $\mathrm{L}$.

* Plumeria alba $\mathrm{L}$.

* Plumeria rubra L.

* Plumeria acutifolia Poir.

Prestonia bahiensis M.Arg.

Prestonia pickelli Maf.

Rauvolfia bahiensis A.DC.

Rauvolfia grandiflora Mart.

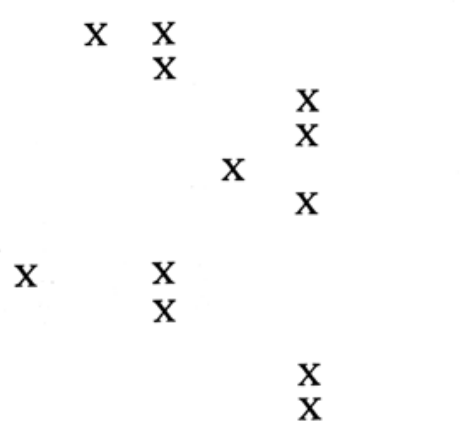
$\mathrm{X}$
$\begin{array}{lllll}X & X & X & \\ & X & & \\ & & & X\end{array}$

Rauvolfia ligustrina $\mathrm{R}$. \& S.

Skytanthus hancorniifolius (DC) Benth.

Tabernaenontava fuschiifolia DC.

*Thevetia neriifolia Juss.

*Thevetia peruviana (Pers.)K.Schum.

$\begin{array}{lll}X & X & \\ & X & X\end{array}$

$\mathrm{X}$ 


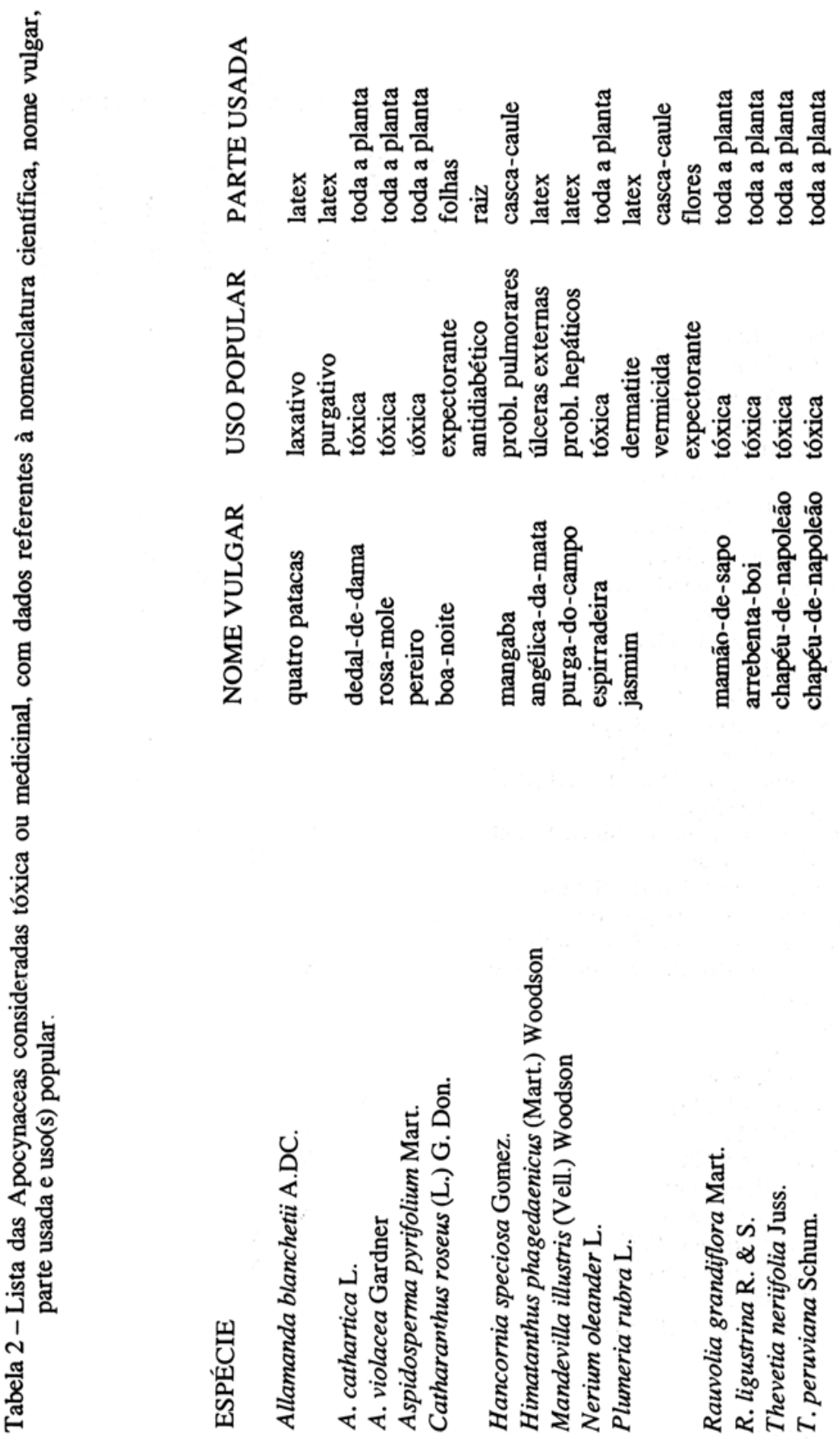




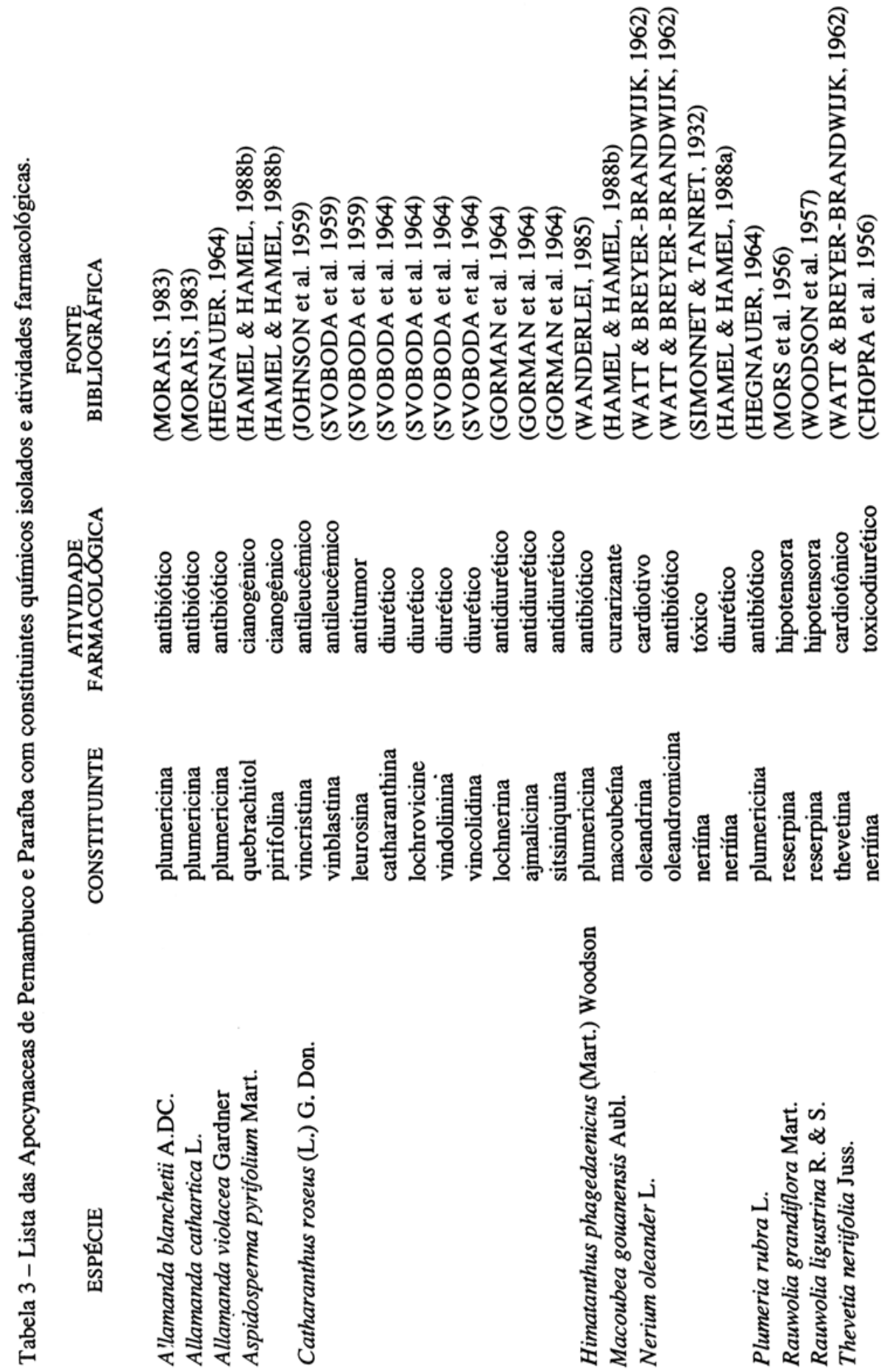

